\title{
Computational approach to identify potential antileishmanial activity of reported inhibitor, E5700 and two natural alkaloids against Leishmania donovani Squalene Synthase
}

\author{
Padmika Wadanambi \\ Department of Parasitology, Faculty of Medicine, University of Colombo, Sri Lanka \\ ${ }^{\star}$ Corresponding author: Email: pwadanambi@gmail.com
}

(Received 01 July 2020; Revised 16 July 2020; Accepted 16 July 2020)

\begin{abstract}
Leishmania species are the causative agents for Leishmaniasis which is one of the neglected tropical diseases causing 70,000 deaths worldwide each year. Squalene synthase enzyme plays a vital role in sterol metabolism which is essential for Leishmania parasite viability. Therefore squalene synthase of Leishmania donovani is a therapeutic target to inhibit growth of parasite. The 3D model of Leishmania donovani Squalene Synthase (LdSQS) was generated by homology modeling and validated through PROCHECK, ERRAT, VERIFY3D and PROSA tools. Virtual screening of the protein was performed by AutoDock with reported inhibitor, E5700 and two natural alkaloids. Molecular interactions were explored to understand the nature of intermolecular bonds between active ligand and the protein binding site residues using UCSF Chimera and PLIP server. The reported inhibitor showed the best binding affinity $(-9.75 \mathrm{kcal} / \mathrm{mol})$ closely followed by Ancistrotanzanine B $(-9.55 \mathrm{kcal} / \mathrm{mol})$ and Holamine $(-8.79 \mathrm{kcal} / \mathrm{mol})$. Ancistrotanzanine B showed low binding energy and permissible ADMET properties. Based on the present study, homology model of LdSQS and Ancistrotanzanine B can be used to design inhibitors with antileishmanial activity.
\end{abstract}

Keywords: Leishmania donovani; Homology Modeling; Molecular Docking; Leishmaniasis; Squalene Synthase

\section{Introduction}

Leishmaniasis is included in the list of WHO as one of the major neglected tropical diseases worldwide (TorresGuerrero et al., 2017). The parasitic disease, Leishmaniasis is transmitted to humans by the bite of a parasiteinfected, female sandfly (Phlebotomus). Mild Cutaneous Leishmaniasis, serious Mucocutaneous Leishmaniasis and fatal Visceral Leishmaniasis are the three clinical forms of the disease. The parasite, Leishmania donovani is the causative agent for Leishmaniasis in Sri Lanka (Karunaweera et al., 2020). However, there is currently no ideal vaccine or a drug of choice available to cure any form of Leishmaniasis. Therefore it is an urgent requirement to investigate new oral drugs that are safe and inexpensive to treat Leishmaniasis.

Targeting sterol metabolism of Leishmania donovani is considered as a therapeutic strategy to inhibit its growth. Among enzymes participating in the sterol biosynthesis pathway, squalene synthase has been identified as a potent drug target to develop anti-parasitic drugs. According to the previous studies, two quinuclidine based inhibitors namely E5700 and ER-119884 have shown inhibitory effects against squalene synthase of Trypanosoma cruzi and Leishmania amazonensis (Fernandes Rodrigues et al., 2008; Urbina et al., 2004). Therefore the inhibition of Leishmania donovani squalene synthase in sterol

\footnotetext{
(c) The Author(s), 2020. Published by Cambridge University Press. This is an Open Access article, distributed under the terms of the Creative Commons Attribution licence (http://creativecommons.org/licenses/by/4.0/), which permits unrestricted re-use, distribution, and reproduction in any medium, provided the original work is properly cited.
} 
(B)

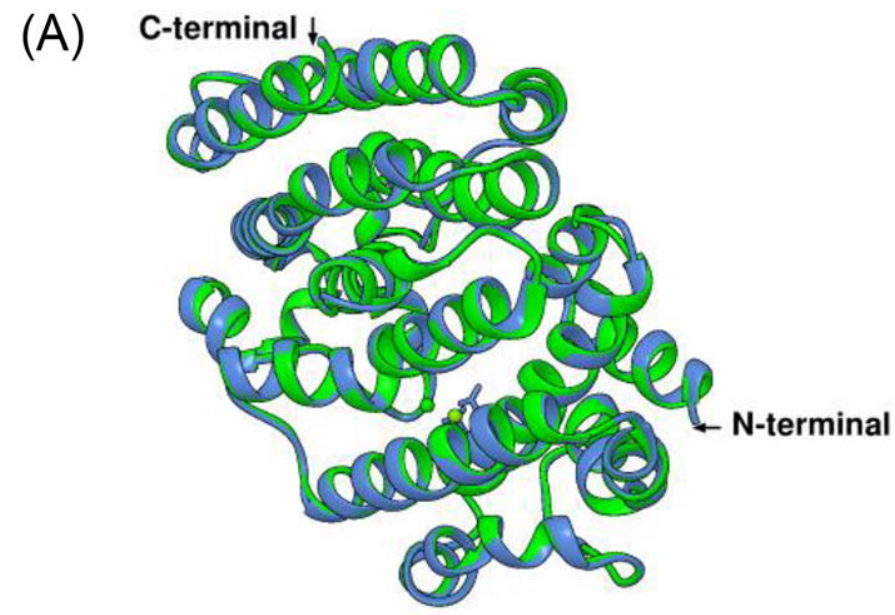

\begin{tabular}{|c|c|}
\hline 3WCA_A & DLRFCYDILQAVSRSFAVVIMEL-DEEMRDAVCIFYLVLRALDTVEDDMSIPVEFKLREL \\
\hline LdSQS & 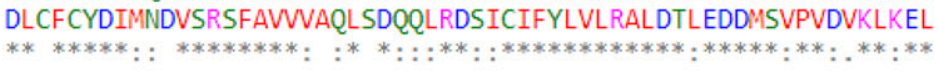 \\
\hline 3WCA_A & PKFHEHLHDTTWCMSGVGVGRERELLERYTHVTRAYSRLGKAYQDVISGICERMANGMCD \\
\hline & 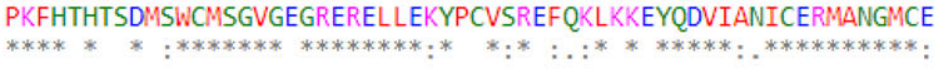 \\
\hline 3WCA_A & FLTRKVETKADYDLYCHYVAGLVGHGLTLLYVSSGLEDVRLADDLTNANHMGLFLQKTNI \\
\hline LdSQS & $\begin{array}{l}\text { FLKRPVITKDDYNQYCHYVAGLVGHGLTQLFARCDFEDPSLDDDLTTSNHMGLFLQKTNI } \\
* * * * * * * * *: * * * * * * * * * * * * * * * * * \ldots . \ldots: * * * * * * * * .: * * * * * * * * * * * * *\end{array}$ \\
\hline 3WCA_A & IRDFYEDICEVPPRVFWPREIWEKYTDDLHAFKDELHEAKAVECLNAMVADALVHVPHVV \\
\hline LdSQS & $\begin{array}{l}\text { IRDYYEDIREEPPRMFWPKEIWGIYVTELKELKSESNNAAAVQCLNAMVADALVHVPYIV } \\
* * *: * * * * * * * *: * * *: * * * *,::^{*}::^{*}, *:: * * *: * * * * * * * * * * * * * * *:: *\end{array}$ \\
\hline 3WCA_A & EYLASLRDPSVFAFSAIPQVMAMATLSLVFNNKDVFHTKVKTTRGATARIFHYSTELQAT \\
\hline LdSQS & 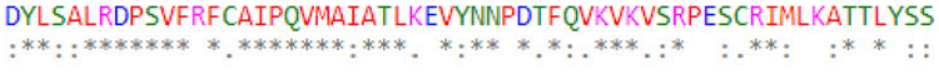 \\
\hline 3WCA_A & LQMLKTYTLRLAARMNAQDACYDRIEHLVNDAIRAME \\
\hline LdSQS & 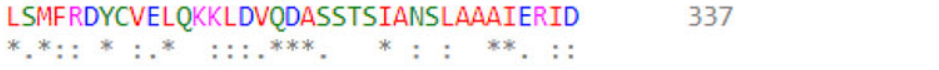 \\
\hline
\end{tabular}

(C)

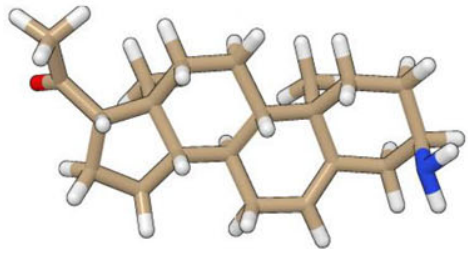

(D)

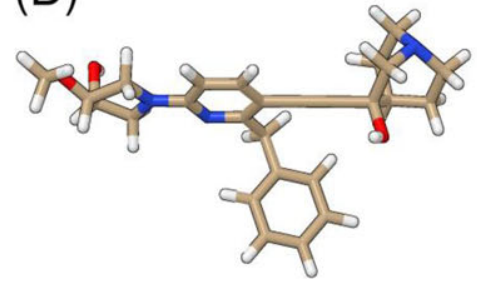

(E)

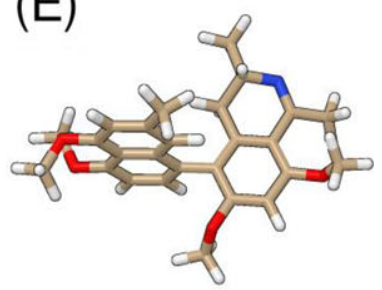

Figure 1. (A). The superimposition of template protein 3WCA_A (Blue) and the 3D modeled structure of LdSQS (Green) $\left(\mathrm{RMSD}=0.306 \mathrm{~A}^{\circ}\right)$. (B). Sequence alignment of LdSQS (Target) with the template protein PDB ID: 3WCA_A. The conserved residues are represented as asterisk, highly similar residues as colon and similar residues as full stop. (C-E). 3D structures of ligands used for the study. [C] Holamine (PubChem CID: 12310548), [D] E5700 (Reported Inhibitor) (PubChem CID: 56947067), [E] Ancistrotanzanine B (PubChem CID: 10002166) 
metabolism could be a promising therapy to prevent the proliferation of parasites due to the collapse of ergosterol production.

Computational study revealed both natural alkaloids interact with active site residues of LdSQS. These compounds could be further evaluated as potent antileishmanial compounds against LdSQS enzyme.

\section{Objective}

In the present study, we attempted to elucidate the three-dimensional structure of Leishmania donovani squalene synthase by homology modeling which is considered to be the most accurate method of structure modeling. The reported inhibitor, E5700 (control) and natural alkaloids were used to discover potential antileishmanial activity against modeled protein by structure-based drug discovery using

Table 1. (A). Identification of the best homologous template using BLAST against Protein Data Bank (PDB). (B). Structure validation results of LdSQS (Modeled Protein). (C). Protein-ligand interaction analysis of docked complexes. (D). Grid box parameters selected for LdSQS (Modeled Protein), based on binding site residues (Spacing was set up at $0.375 \mathrm{~A}^{\circ}$ ). (E). Drug likeness and in silico ADMET (Absorption, Distribution, Metabolism, Excretion and Toxicity) prediction of ligands.

\begin{tabular}{|c|c|c|c|c|c|c|c|c|}
\hline $\begin{array}{l}\text { (A) PDB } \\
\text { ID }\end{array}$ & $\begin{array}{l}\text { Name of } \\
\text { Protein }\end{array}$ & Source & Chain & $\begin{array}{l}\% \\
\text { Identity }\end{array}$ & $\begin{array}{l}\text { Query } \\
\text { Cover }\end{array}$ & $\begin{array}{l}\mathrm{E} \\
\text { value }\end{array}$ & $\begin{array}{l}\text { Max } \\
\text { Score }\end{array}$ & $\begin{array}{l}\text { Total } \\
\text { Score }\end{array}$ \\
\hline 3WCA & $\begin{array}{l}\text { Farnesyltransfera } \\
\text {-se,Putative }\end{array}$ & $\begin{array}{c}\text { Trypanosoma } \\
\text { cruzi strain } \\
\text { CL Brener }\end{array}$ & A & 61.42 & $100 \%$ & $4 e-158$ & 438 & 438 \\
\hline \multicolumn{3}{|c|}{ (B) Description } & & & \multicolumn{3}{|c|}{ LdSQS (Modeled Protein) } & Preferred \\
\hline \multicolumn{9}{|c|}{ Ramachandran plot (PROCHECK) } \\
\hline \multicolumn{5}{|c|}{ Favoured regions (\%) } & \multicolumn{3}{|l|}{94.6} & $>90$ \\
\hline \multicolumn{5}{|c|}{ Allowed regions (\%) } & \multicolumn{4}{|l|}{5.1} \\
\hline \multicolumn{5}{|c|}{ Generously allowed regions (\%) } & \multicolumn{4}{|l|}{0.3} \\
\hline \multicolumn{5}{|c|}{ Clashscore (MolProbity) } & \multicolumn{3}{|c|}{$76^{\text {th }}$ percentile } & percentile $>=66$ \\
\hline \multicolumn{5}{|c|}{ Poor rotamers (\%) (MolProbity) } & \multicolumn{3}{|l|}{0} & $<0.3$ \\
\hline \multicolumn{5}{|c|}{ Favoured rotamers (\%) (MolProbity) } & \multicolumn{3}{|l|}{98.03} & $>98$ \\
\hline \multicolumn{5}{|c|}{ Percentage of the amino acids have scored } & 91.69 & & & $>80$ \\
\hline \multicolumn{9}{|c|}{$>=0.2$ in the 3D/1D profile (VERIFY3D) } \\
\hline \multicolumn{5}{|c|}{ Quality factor (\%) (ERRAT) } & \multicolumn{3}{|c|}{94.8328} & $>=95$ \\
\hline \multicolumn{5}{|c|}{$\mathrm{RMSD}^{*}\left(\mathrm{~A}^{\circ}\right)$ (Superimposition of the modeled Protein with the template) } & \multicolumn{3}{|l|}{0.306} & $<=2.0$ \\
\hline (C) No. & Docked Complex & $\mathrm{LBE}^{\star}(\mathrm{kcal} / \mathrm{mol})$ & \multicolumn{2}{|c|}{$\mathrm{EIC}^{\star *}(\mathrm{nM})$} & \multicolumn{4}{|c|}{ Interacting residues } \\
\hline \multirow[t]{8}{*}{1} & LdSQS-E5700 & -9.75 & 71.84 & & \multicolumn{4}{|c|}{ Hydrogen Bonds } \\
\hline & & & & & Val139, & /s255, Gl & & \\
\hline & & & & & Hydrop & bic Inte & tions & \\
\hline & & & & & Phe16, & e35, Leı & , Ala14 & Val143, Leu175 \\
\hline & & & & & $\mathrm{Pi}-\mathrm{Sta}$ & ing & & \\
\hline & & & & & Tyr36 & & & \\
\hline & & & & & Salt Bri & & & \\
\hline & & & & & Asp43 & & & \\
\hline 2 & LdSQS-Ancis & -9.55 & 99.59 & & Hydrop & bic Inte & tions & \\
\hline & trotanzanine B & & & & $\begin{array}{r}\text { Phe16, } \\
\text { Leu1 }\end{array}$ & $\begin{array}{l}\text { I19, Phe } \\
\text { Phe254 }\end{array}$ & $\begin{array}{l}\text { Tyr36, } \\
\text { ro258 }\end{array}$ & al143, Leu147, \\
\hline
\end{tabular}


Table 1 Continued

\begin{tabular}{|c|c|c|c|c|c|c|}
\hline (C) No. & Docked Complex & $\mathrm{LBE}^{\star}(\mathrm{kcal} / \mathrm{mol})$ & $\mathrm{EIC}^{\star \star}(\mathrm{nM})$ & \multicolumn{3}{|c|}{ Interacting residues } \\
\hline 3 & LdSQS-Holamine & -8.79 & 362.95 & \multicolumn{3}{|c|}{ Hydrogen Bonds } \\
\hline & & & & \multicolumn{3}{|c|}{ Val139, Gln 259} \\
\hline & & & & \multicolumn{3}{|c|}{ Hydrophobic Interactions } \\
\hline & & & & \multicolumn{3}{|c|}{$\begin{array}{l}\text { Phe16, Tyr36, Ala140, Val143, Leu147, Leu175, } \\
\text { Phe254, Pro258 }\end{array}$} \\
\hline $\begin{array}{l}\text { (D) Targ } \\
\text { Protein }\end{array}$ & \multicolumn{2}{|c|}{ Binding Site Residues } & \multicolumn{2}{|c|}{$\begin{array}{l}\text { Centre Grid Box (Points in X, Y, Z - } \\
\text { Axis) }\end{array}$} & \multicolumn{2}{|c|}{$\begin{array}{l}\text { Size (Points in } X, Y, Z \text { - } \\
\text { Axis) }\end{array}$} \\
\hline \multirow[t]{7}{*}{ LdSQS } & \multicolumn{2}{|c|}{ Phe16, Val20, Ile32, Phe35, } & \multicolumn{2}{|c|}{$68.920 \times 7.800 \times 62.600$} & \multicolumn{2}{|c|}{$40 \times 40 \times 58$} \\
\hline & \multicolumn{6}{|c|}{ Tyr36, Leu39, Arg40, Asp43, } \\
\hline & \multicolumn{6}{|c|}{ Val139, Ala140, Val143, Gly144 } \\
\hline & \multicolumn{6}{|c|}{$\begin{array}{l}\text { Leu147, Thr148, Phe151, } \\
\text { Ser168 }\end{array}$} \\
\hline & \multicolumn{6}{|c|}{$\begin{array}{l}\text { Met171, Gly172, Leu175, } \\
\text { Gln176 }\end{array}$} \\
\hline & \multicolumn{6}{|c|}{$\begin{array}{l}\text { Tyr242, Phe254, Cys } 255 \\
\text { Pro258 }\end{array}$} \\
\hline & \multicolumn{5}{|l|}{ Gln259 } & \\
\hline \multicolumn{2}{|c|}{ (E) Description } & Preferred & E5700 & \multicolumn{2}{|c|}{ Ancistrotanzanine B } & Holamine \\
\hline \multicolumn{2}{|c|}{ Drug Likeness } & - & Suitable & Suitable & & Suitable \\
\hline \multicolumn{7}{|c|}{ Absorption } \\
\hline \multicolumn{2}{|c|}{ Human Intestinal Absorption (\%) } & $>30$ & 97.491 & 98.598 & & 94.162 \\
\hline \multicolumn{7}{|c|}{ Distribution } \\
\hline \multicolumn{2}{|c|}{ VDss* $^{*}[\log (\mathrm{L} / \mathrm{kg})]$} & $>0.45$ & 0.959 & 0.441 & & 0.476 \\
\hline \multicolumn{2}{|c|}{$\mathrm{BBB}^{\star \star}$ permeability $(\log \mathrm{BB})$} & -1.0 & -0.51 & -0.527 & & 0.049 \\
\hline \multicolumn{7}{|c|}{ Metabolism } \\
\hline \multicolumn{2}{|c|}{ CYP2D6 substrate ${ }^{\star \star \star}$} & - & No & No & & No \\
\hline CYP3A4 & ubstrate ${ }^{\star \star \star}$ & - & Yes & Yes & & Yes \\
\hline CYP1A2 & hibitor ${ }^{\star \star \star}$ & - & No & Yes & & No \\
\hline $\mathrm{CYP} 2 \mathrm{C} 1$ & inhibitor $^{\star \star \star}$ & - & No & Yes & & No \\
\hline CYP2C9 & hibitor ${ }^{\star \star \star}$ & - & No & Yes & & No \\
\hline CYP2D6 & hibitor ${ }^{\star \star *}$ & - & No & No & & No \\
\hline CYP3A4 & hibitor ${ }^{\star \star \star}$ & - & Yes & Yes & & No \\
\hline Excreti & & & & & & \\
\hline Total cl & rance $[\log (\mathrm{ml} / \mathrm{min} / \mathrm{kg})]$ & $<1.28$ & 0.702 & 0.505 & & 0.303 \\
\hline Toxicit & & & & & & \\
\hline AMES t & icity & - & No & No & & No \\
\hline Hepato & xicity & - & Yes & No & & Yes \\
\hline
\end{tabular}

RMSD* - Root Mean Square Deviation

$\mathrm{LBE}^{\star}$ - Lowest Binding Energy (kcal/mol), EIC** - Estimated Inhibition constant (Ki)(nM)

VDss $^{\star}$ - Volume of Distribution Human, BBB ${ }^{\star \star}$ - Blood Brain Barrier

CYP $^{\star \star *}$ - Cytochrome P450 Enzymes 
(A)

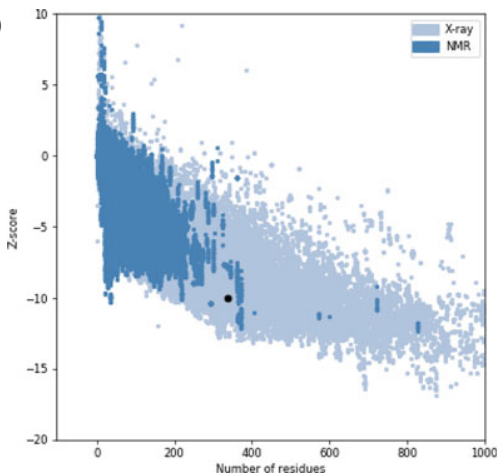

(B)

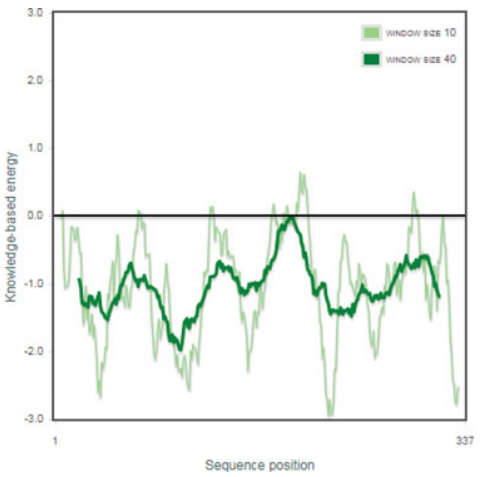

(C) PROCHECK

Ramachandran Plot

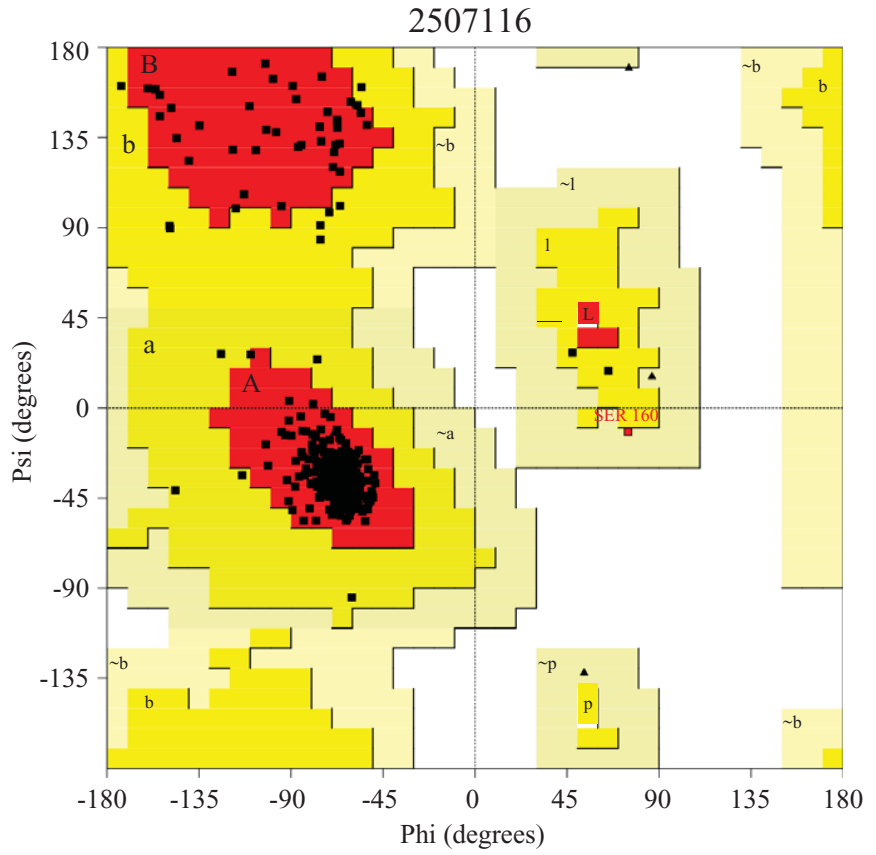

Plot statistics

$\begin{array}{lrr}\text { Residues in most favoured regions [A,B,L] } & 296 & 94.6 \% \\ \text { Residues in additional allowed regions [a,b,l,p] } & 16 & 5.1 \% \\ \text { Residues in generously allowed regions [ a, } \sim \mathrm{b}, \sim 1, \sim \mathrm{p}] & 1 & 0.3 \% \\ \text { Residues in disallowed regions } & 0 & 0.0 \% \\ & ---\cdot & \\ \text { Number of non-glycine and non-proline residues } & 313 & 100.0 \% \\ \text { Number of end-residues (excl. Gly and Pro) } & 3 & \\ \text { Number of glycine residues (shown as triangles) } & 9 & \\ \text { Number of proline residues } & 13 & \\ \text { Total number of residues } & ---. & \end{array}$

Figure 2. (A). ProSA z-score plot of the LdSQS protein. The z-score indicates overall model quality. The z-score value (-10.03) of LdSQS falls in the range of PDB proteins submitted by X ray crystallography (Light Blue), indicating a good quality model. (B). ProSA energy profile plot of the LdSQS protein. The plot shows local model quality by plotting energies as a function of amino acid sequence. The ProSA analysis of the model shows maximum residues in the negative energy region, indicating a reliable and good quality structure. (C). Ramachandran plot of the LdSQS protein built by PROCHECK. The red regions represent the most favorable regions of residues, the yellow areas represent additionally allowed regions, the light yellow regions are generously allowed regions and the white fields represent the disallowed regions. (D). ERRAT plot for the LdSQS protein. Black bars depict the error region above $99 \%$, gray bars indicate the error region between $95 \% \& 99 \%$ and white bars demonstrate the error region below $95 \%$. The maximum residues of LdSQS are located below $95 \%$ error region. The overall quality factor (94.833) of LdSQS indicates a high resolution structure. (E). VERIFY 3D plot of LdSQS protein. The plot predicts $91.69 \%$ of the residues in LdSQS have an averaged 3D-1D score $>=0.2$, verifying the model. 
(D) Program: ERRAT2

File: LdSQS.pdb

Overall quality factor ${ }^{* *}: 94.833$

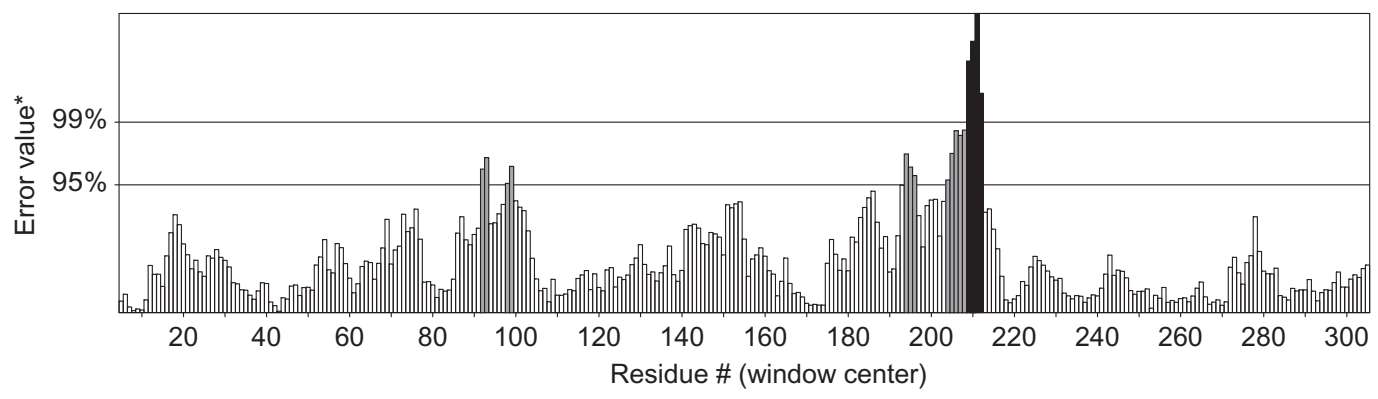

(E)

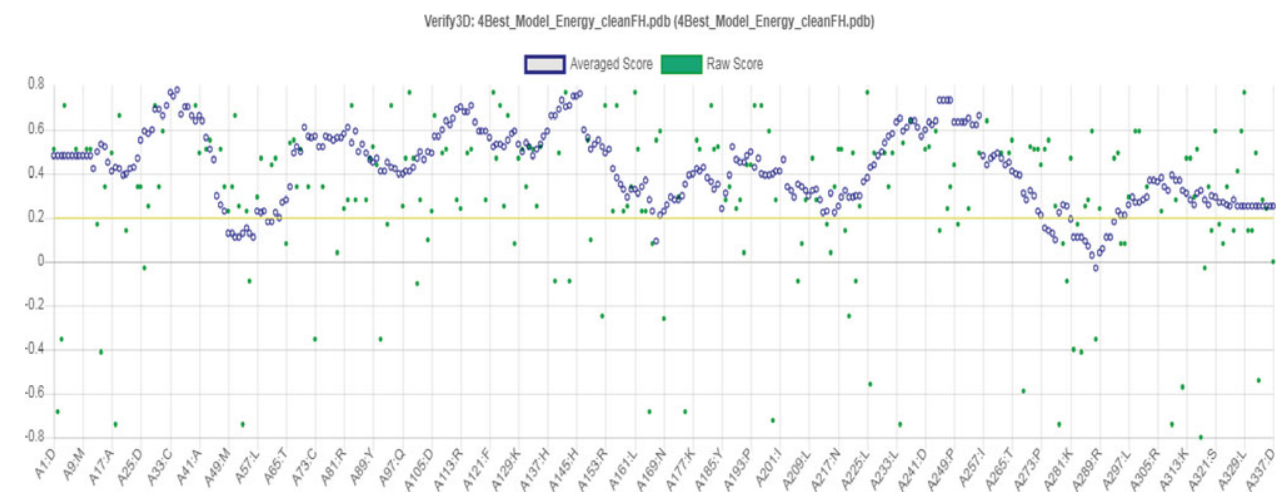

Figure 2. Continued

AutoDock software (Morris et al., 2009). This kind of computer-aided drug design method is useful to reduce the burden of laborious work and money wastage in drug development.

\section{Methods}

The 3D structure of LdSQS was not publicly available in the RCSB Protein Data Bank (https://www. rcsb.org/). Therefore the amino acid sequence of LdSQS (ID: Q257D4) was retrieved from UniProtKB database (https://www.uniprot.org/). The best homologous template was identified by BLASTp. Both C and $\mathrm{N}$ termini, segments of LdSQS which are lack of matching template residues were removed. The final optimal alignment was carried out in Clustal Omega server (Sievers \& Higgins, 2017). Totally 50 models of LdSQS were generated using Modeller v9.22 (Eswar et al., 2006). Out of 50 models, the best model was selected based on the least DOPE score. Further refinement of selected 3D model was performed in the UCSF Chimera v.1.12 (Pettersen et al., 2004) using quality information from MolProbity server (http:// molprobity.biochem.duke.edu/) (Chen et al., 2009). Final energy minimization (1000 steps Steepest Descent in AMBER force field) was continued to remove clashes. Eventually the refined model was subjected to quality validation using SAVES v5.0 server (https://servicesn.mbi.ucla.edu/SAVES/) and ProSA server (Weiderstein \& Sippl, 2007). The best predicted model was executed for virtual screening. All ligands (Figure 1(C)) except E5700 (reported inhibitor taken from PDB ID: 3WCC) were fetched by PubChem CID into UCSF Chimera. Then hydrogens and gastieger charges were added to all ligands. The binding site residues were determined by redocking of template inhibitor (PDB ID: 3WCC) with the modeled protein. The grid box parameters and binding site residues selected for LdSQS are shown in Table 1(D). Molecular simulation was carried out by AutoDock 4.2 using default parameters. The 


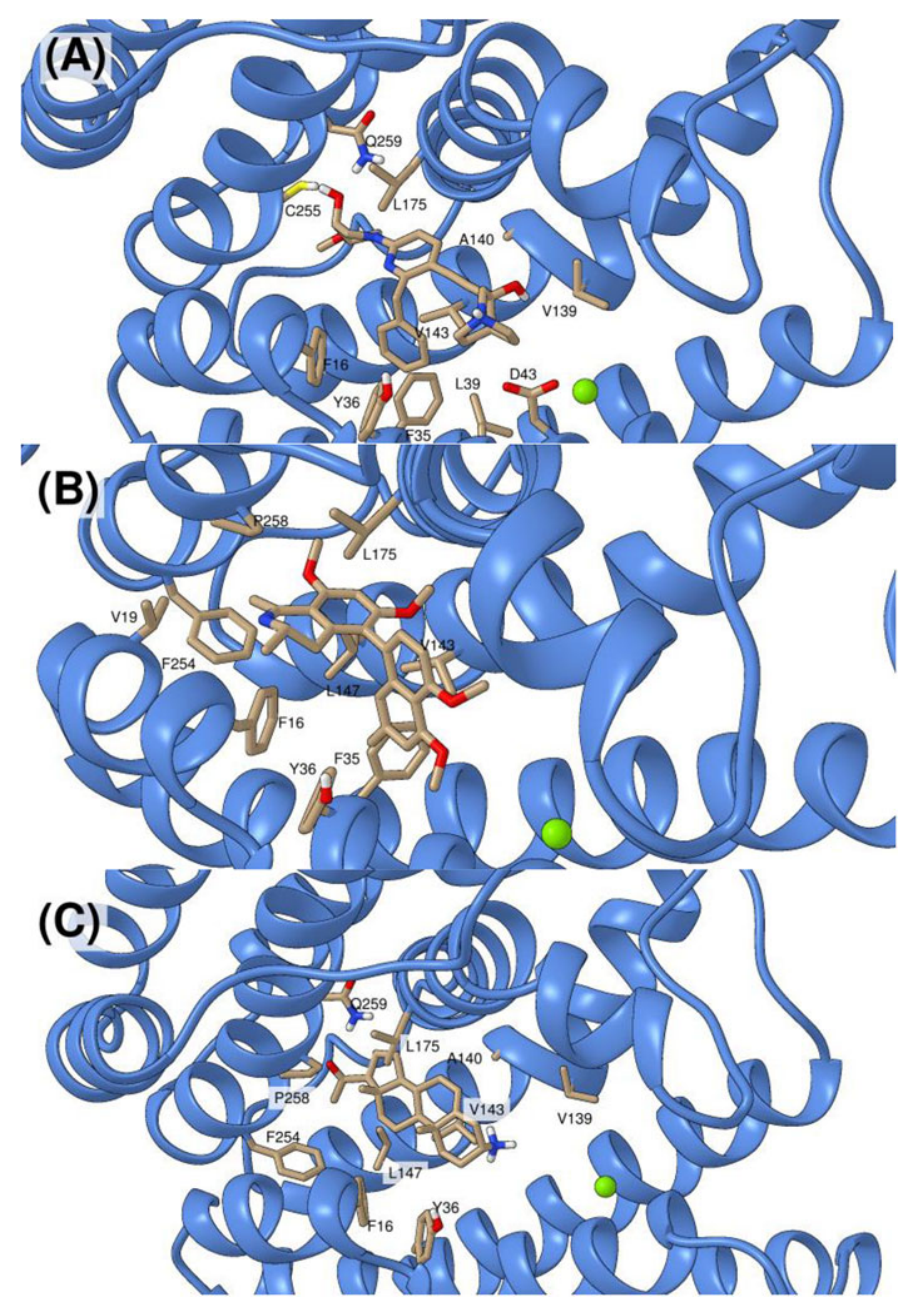

Figure $3(\mathrm{~A}-\mathrm{C})$. Best binding conformation of ligand with LdSQS protein in the binding cavity. These images were generated in UCSF Chimera. (A). Best binding conformation of E5700 (Reported Inhibitor) to LdSQS. (B). Best binding conformation of Ancistrotanzanine B to LdSQS. (C). Best binding conformation of Holamine to LdSQS

interactions of docked complexes were analyzed using PLIP server (https://projects.biotec.tu-dresden.de/ plip-web/plip) (Salentin et al., 2015) and visualized in UCSF Chimera. The pkCSM server (http://biosig. unimelb.edu.au/pkcsm/) (Pires et al., 2015) was used to ADMET analysis of all ligands.

\section{Results}

The BLASTp search identified PDB ID: 3WCA (A chain) as the best homologous template for homology modeling (Table 1(A)). The pairwise alignment (Figure 1(B)) and superimposition of template with final LdSQS model (Figure $1(\mathrm{~A})$ ) shows $61.42 \%$ sequence similarity and $0.306 \mathrm{~A}^{\circ}$ RMSD respectively. The quality validation of the LdSQS model evaluated via PROCHECK, ERRAT, VERIFY 3D and ProSA, is shown in Table $1(B)$ \& Figure 2(A-E). The validation results prove LdSQS as a good quality model. Following docking simulations, the highest affinity towards LdSQS was observed for E5700 (-9.75 kcal/ $\mathrm{mol})$ closely followed by Ancistrotanzanine B $(-9.55 \mathrm{kcal} / \mathrm{mol})$ and Holamine $(-8.79 \mathrm{kcal} / \mathrm{mol}) \mathrm{respec}-$ tively. The reported inhibitor formed three hydrogen bonds with the key residues Val139, Cys255 and 
Gln259 of LdSQS while Holamine bound to Val139 and Gln259 through hydrogen bond interactions. The best docking alkaloid, Ancistrotanzanine B was stabilized only by hydrophobic interactions. The best binding conformation of each ligand is depicted in Figure 3. All ligands (Figure 1(C)) satisfied Lipinski's rule of five which is essential for Drug likeness. According to ADMET predictions (Table 1(E)) except Ancistrotanzanine B, all show hepatotoxicity.

\section{Discussions}

Due to high sequence identity, query coverage and structure completeness, a single template was selected for modeling of LdSQS. All validation results including ERRAT, PROCHECK, VERIFY3D, ProSA and RMSD were in the acceptable ranges verifying a high quality model. The negative and low binding energy of docked complex demonstrates the strong affinity between LdSQS and the ligand occupying the most favorable conformation. Binding energy of the reported inhibitor (-9.75) was superior to natural alkaloids (-9.55 \& -8.79), showing the strongest affinity. Previous studies have reported E5700 (Fernandes Rodrigues et al., 2008) as an anti-proliferative agent against squalene synthase of Leishmania amazonensis. For the reported inhibitor, experimental data revealed MIC values $30 \mathrm{nM}$ for NR strain and $50 \mathrm{nM}$ for Josefa strain of Leishmania amazonensis promastigotes. The inhibitory effect of E5700 was also confirmed by current docking studies. Interestingly, Ancistrotanzanine B showed binding energy close to E5700 and stabilized only by hydrophobic interactions to LdSQS showing no potential risk of hepatotoxicity.

\section{Conclusions}

The 3D structure of LdSQS was evaluated by homology modeling. The generated model was validated stereochemically and structurally by different tools to obtain a good quality model. Then molecular docking was carried out to find binding affinity and molecular interactions between LdSQS and ligands. Virtual screening revealed that the most interacting binding site residues of LdSQS are Phe16, Val19, Phe35, Tyr36, Leu39, Asp43, Val139, Ala140, Val143, Leu147, Leu175, Phe254, Cys255, Pro258 and Gln259.Both natural alkaloids showed close binding affinity compared to reported inhibitor and admissible ADME profile except hepatotoxicity for Holamine. Therefore homology model of LdSQS and natural alkaloids can be further structurally optimized to design potent inhibitors with antileishmanial activity.

Acknowledgements. The author would like to thank Prof. Nadira Karunaweera, Department of Parasitology, Faculty of Medicine, University of Colombo, Sri Lanka, for the help and support.

Author Contributions. I confirm that I carried out all the work and no other co-worker was involved. The author read and approved the final manuscript.

Funding Information. This research received no specific grant from any funding agency, commercial or not - for- profit sectors.

Conflict of Interest. The author declares no conflict of interest.

Data Availability. The author confirms that the data supporting the findings of this study are available within the article.

\section{References}

Chen, V. B., Arendall, W. B., Headd, J. J., Keedy, D. A., Immormino, R. M., Kapral, G. J., Murray, L. W., Richardson, J. S., \& Richardson, D. C. (2009). MolProbity: All-atom structure validation for macromolecular crystallography. Acta Crystallographica Section D Biological Crystallography, 66, 12-21. https://doi.org/10.1107/s0907444909042073.

Eswar, N., Webb, B., Marti-Renom, M. A., Madhusudhan, M. S., Eramian, D., Shen, M., Pieper, U., \& Sali, A. (2006). Comparative protein structure modeling using modeller. Current Protocols in Bioinformatics, 15, 5.6.1-5.6.30. https:// doi.org/10.1002/0471250953.bi0506s15. 
Fernandes Rodrigues, J. C., Concepcion, J. L., Rodrigues, C., Caldera, A., Urbina, J. A., \& deSouza, W. (2008). In vitro activities of ER-119884 and E5700, two potent squalene synthase inhibitors, against Leishmania amazonensis: Antiproliferative, biochemical, and ultrastructural effects. Antimicrobial Agents and Chemotherapy, 52, 4098-4114. https://doi.org/ 10.1128/aac.01616-07

Karunaweera, N. D., Ginige, S., Senanayake, S., Silva, H., Manamperi, N., Samaranayake, N., Siriwardana, Y., Gamage, D., Senerath, U., \& Zhou, G. (2020). Spatial epidemiologic trends and hotspots of Leishmaniasis, Sri Lanka, 2001-2018. Emerging Infectious Diseases, 26, 1-10. https://doi.org/10.3201/eid2601.190971.

Morris, G. M., Huey, R., Lindstrom, W., Sanner, M. F., Belew, R. K., Goodsell, D. S., \& Olson, A. J. (2009). AutoDock4 and AutoDockTools4: Automated docking with selective receptor flexibility. Journal of Computational Chemistry, 30, 2785-2791. https://doi.org/10.1002/jcc.21256.

Pettersen, E. F., Goddard, T. D., Huang, C. C., Couch, G. S., Greenblatt, D. M., Meng, E. C., \& Ferrin, T. E. (2004). UCSF Chimera? A visualization system for exploratory research and analysis. Journal of Computational Chemistry, 25, 1605-1612. https://doi.org/10.1002/jcc.20084.

Pires, D. E. V., Blundell, T. L., \& Ascher, D. B. (2015). pkCSM: Predicting small-molecule pharmacokinetic and toxicity properties using graph-based signatures. Journal of Medicinal Chemistry, 58, 4066-4072. https://doi.org/10.1021/acs. jmedchem.5b00104.

Salentin, S., Schreiber, S., Haupt, V. J., Adasme, M. F., \& Schroeder, M. (2015). PLIP: Fully automated protein-ligand interaction profiler. Nucleic Acids Research, 43, W443-W447. https://doi.org/10.1093/nar/gkv315.

Sievers, F., \& Higgins, D. G. (2017). Clustal Omega for making accurate alignments of many protein sequences. Protein Science, 27, 135-145. https://doi.org/10.1002/pro.3290.

Torres-Guerrero, E., Quintanilla-Cedillo, M. R., Ruiz-Esmenjaud, J., \& Arenas, R. (2017). Leishmaniasis: A review. F1000Research, 6, 750. https://doi.org/10.12688/f1000research.11120.1.

Urbina, J. A., Concepcion, J. L., Caldera, A., Payares, G., Sanoja, C., Otomo, T., \& Hiyoshi, H. (2004). In vitro and in vivo activities of E5700 and ER-119884, two novel orally active squalene synthase inhibitors, against Trypanosoma cruzi. Antimicrobial Agents and Chemotherapy, 48, 2379-2387. https://doi.org/10.1128/aac.48.7.2379-2387.2004.

Wiederstein, M., \& Sippl, M. J. (2007). ProSA-web: Interactive web service for the recognition of errors in three-dimensional structures of proteins. Nucleic Acids Research, 35, W407-W410. https://doi.org/10.1093/nar/gkm290.

Cite this article: Wadanambi P (2020). Computational approach to identify potential antileishmanial activity of reported inhibitor, E5700 and two natural alkaloids against Leishmania donovani Squalene Synthase Experimental Results, 1, e31, 1-13. https://doi.org/10.1017/exp.2020.37 


\section{Peer Reviews}

\section{Reviewing editor: Dr. Emanuele Frontoni}

Universita Politecnica delle Marche, Information Engineerging Department - DII, Ancona, Italy, 60121

This article has been accepted because it is deemed to be scientifically sound, has the correct controls, has appropriate methodology and is statistically valid, and met required revisions.

doi:10.1017/exp.2020.37.pr1

Review 1: Computational approach to identify potential antileishmanial activity of reported inhibitor, E5700 and two natural alkaloids against Leishmania donovani Squalene Synthase

Reviewer: Dr. Sara Moccia

Università Politecnica delle Marche, Department of Information Engineering, Ancona, Italy

Date of review: 07 July 2020

(C) The Author(s), 2020. Published by Cambridge University Press This is an Open Access article, distributed under the terms of the Creative Commons Attribution licence (http://creativecommons.org/licenses/by/4.0/), which permits unrestricted re-use, distribution, and reproduction in any medium, provided the original work is properly cited.

Conflict of interest statement. Reviewer declares none

Comments to the Author: A more in depth description of limitations and future work is required. Larger space should be given to the survey of the state of the art.

Proofreading is required (sometimes, there is no space between words)

\section{Score Card}

Presentation

2.7

Is the article written in clear and proper English? (30\%)

Is the data presented in the most useful manner? (40\%)

Does the paper cite relevant and related articles appropriately? (30\%)

Context

Does the title suitably represent the article? (25\%)

Does the abstract correctly embody the content of the article? (25\%)

Does the introduction give appropriate context? (25\%)

Is the objective of the experiment clearly defined? (25\%) 
Analysis

2.8

Does the discussion adequately interpret the results presented? (40\%)

Is the conclusion consistent with the results and discussion? (40\%)

Are the limitations of the experiment as well as the contributions of the experiment clearly outlined? (20\%) 
Review 2: Computational approach to identify potential antileishmanial activity of reported inhibitor, E5700 and two natural alkaloids against Leishmania donovani Squalene Synthase

Reviewer: Dr. Marta Branquinha

UFRJ, Microbiologia Geral, Rio de Janeiro, RJ, Brazil

Date of review: 06 July 2020

(C) The Author(s), 2020. Published by Cambridge University Press This is an Open Access article, distributed under the terms of the Creative Commons Attribution licence (http://creativecommons.org/licenses/by/4.0/), which permits unrestricted re-use, distribution, and reproduction in any medium, provided the original work is properly cited.

Conflict of interest statement. Reviewer declares none.

Comments to the Author: This is an interesting topic: the homology modeling of squalene synthase to search for the antileishmanial activity of different molecules by structure-based drug discovery. It is my opinion that the paper deserves publication in Experimental Results. However, the manuscript does not read fluently: some mistakes were found throughout the text and must be corrected.

\section{Score Card}

Presentation

Is the article written in clear and proper English? (30\%)

Is the data presented in the most useful manner? $(40 \%)$

Does the paper cite relevant and related articles appropriately? (30\%)

Context

Does the title suitably represent the article? (25\%)

Does the abstract correctly embody the content of the article? (25\%)

Does the introduction give appropriate context? (25\%)

Is the objective of the experiment clearly defined? (25\%)

Analysis

Are the limitations of the experiment as well as the contributions of the experiment clearly outlined? (20\%) 
Review 3: Computational approach to identify potential antileishmanial activity of reported inhibitor, E5700 and two natural alkaloids against Leishmania donovani Squalene Synthase

Reviewer: Dr. Leandro Stefano Sangenito (D)

UFRJ, Instituto de Microbiologia, Rio de Janeiro, Brazil, 21941-901

Date of review: 08 July 2020

Conflict of interest statement. Reviewer declares none

Comments to the Author: The present work was well conducted by the author and represents an addition when the proposal is to develop new methods to combat leishmaniasis.

Please see spacing errors between words and punctuation.

I understand the proposal of both the present work and Experimental Results but I missed more information in the discussion. As a suggestion, for example, in addition to the computational field, the author could correlate his data with the results in the literature with squalene synthase inhibitors in leishmania. There are some studies with E5700, ER-119884 and other inhibitors in leishmania in in vitro models. This correlation could further corroborate the author's assumptions.

\section{Score Card}

Presentation

Is the data presented in the most useful manner? (40\%)

Does the paper cite relevant and related articles appropriately? (30\%)

Context

5.0

Does the title suitably represent the article? (25\%)

Does the abstract correctly embody the content of the article? (25\%)

Does the introduction give appropriate context? (25\%)

Is the objective of the experiment clearly defined? (25\%)

Analysis

Are the limitations of the experiment as well as the contributions of the experiment clearly outlined? (20\%) 